\title{
Performance Evaluation of Communication Software Systems for Distributed Computing
}

\author{
Rod Fatoohi*
}

Report NAS-96-006, June 1996

\begin{abstract}
In recent years there has been an increasing interest in object-oriented distributed computing since it is better quipped to deal with complex systems while providing extensibility, maintainability, and reusability. At the same time, several new high-speed network technologies have emerged for local and wide area networks. However, the performance of networking software is not improving as fast as the networking hardware and the workstation microprocessors. This paper gives an overview and evaluates the performance of the Common Object Request Broker Architecture (CORBA) standard in a distributed computing environment at NASA Ames Research Center. The environment consists of two testbeds of SGI workstations connected by four networks: Ethernet, FDDI, HiPPI, and ATM. The performance results for three communication software systems are presented, analyzed and compared. These systems are: BSD socket programming interface, IONA's Orbix, an implementation of the CORBA specification, and the PVM message passing library. The results show that high-level communication interfaces, such as CORBA and PVM, can achieve reasonable performance under certain conditions.
\end{abstract}

*The author is an employee of MRJ, Inc. Mailing address: Rod Fatoohi, Mail Stop T27A-1, NASA Ames Research Center, Moffett Field, CA 94035, Ph. (415) 604-3486, Fax. (415) 604-3957, E-mail: fatoohi@nas.nasa.gov. This work was funded through NASA contract NAS 2-14303. 



\section{Introduction}

One of the most interesting emerging technologies is Object-Oriented distributed computing. Current technology based on systems like the Distributed Computing Environment (DCE) and using the Remote Procedure Call (RPC) mechanisms do not provide adequate support for building a real, complex, scalable, distributed computing environment. For example, RPC does not provide explicit support for moving objects nor does it handle distributed objects in a transparent manner. Also, despite the fact that RPC is an important part of most distributed systems including the objected-oriented systems, it is still a relatively low level interface to the distributed computing environment. The Common Object Request Broker Architecture (CORBA) specification provides the mechanisms for objects to interact transparently.

Another emerging technology is high-speed networks. Emerging networks have peak transmission rates of about one hundred megabytes per second. Combined with high connectivity (switched network), the aggregate network bandwidth can reach several gigabytes per second.

The distributed object technology along with high-speed networks represent two key components of the information infrastructure for next generation applications. The object-oriented technology provides the extensibility, maintainability, and reusability that are needed in software engineering while the high-speed network technology provides the required speed to transmit information between systems. These technologies are essential in many applications such as multimedia, virtual manufacturing, digital library, air traffic control simulation, and virtual computing. At this time, it is important to evaluate these technologies and identify their limitations since some products, based on these technologies, have started to appear in the commercial market.

One of the few studies of the performance of CORBA on high-speed networks is the work by Schmidt et al. [9] where they compared the performance of BSD sockets and two implementations of CORBA (Orbix from IONA Technologies and ORBeline from Post Modern Computing) over Ethernet and ATM networks using two SPARCstations. They showed that CORBA based implementations of $t$ tcp were slower than BSD sockets implementations. Also, they reported good results using the ACE toolkit.

This is the first step in evaluating distributed systems and analyzing different components of these systems. Here the emphasis is on examining the performance of communication software systems under different conditions and identifying factors that influence their performance. The communication tests were chosen to study such factors as: message size, socket buffer size, and data type. Two communication systems are considered here: Orbix, an implementation of the CORBA specification, and the PVM message passing library. The performance results from these two systems are compared to the results obtained from using the BSD socket programming interface.

There are many similarities and differences between CORBA and PVM. Both CORBA and PVM are based on the BSD socket programming interface. Also, both systems consist of communication libraries and a run time system (daemon). In addition, both systems can be configured to run on a single processor, for testing and 
debugging, as well as on heterogeneous platforms of different machines and networks. Moreover, both systems can be used as high-level network programming interfaces for building a large distributed system. Finally, both systems provide similar functionalities in moving data, except that PVM is richer in group communication.

The main differences between PVM and CORBA is that PVM, like DCE, is based on a procedure-oriented distribution model while CORBA is based on an objectoriented distribution model. Also, PVM was designed for networks of workstations as well as parallel computers while CORBA was designed for distributed computing using a client/server model. Both systems, with certain modifications and added services, can work in both environments. Despite these differences, a study of these systems would give an insight of the performance issues that users might face.

Experiments were performed on two testbeds consisting of Silicon Graphics, Inc. (SGI) workstations connected by four networks at the Numerical Aerodynamic Simulation (NAS) Systems division at NASA Ames Research Center. These networks are: a $10 \mathrm{Mbits} / \mathrm{s}$ Ethernet, a $100 \mathrm{Mbits} / \mathrm{s}$ FDDI, an $800 \mathrm{Mbits} / \mathrm{s}$ HiPPI, and a 155 Mbits/s ATM.

The paper starts with an overview of CORBA and PVM. Followed by a brief description of the hardware platforms and the networks. Next, the performance results for the three communication software systems are presented and modeled. Finally, we offer some concluding remarks.

\section{CORBA}

The Common Object Request Broker Architecture (CORBA) is a standard for transparent communication between application objects being developed by the Object Management Group (OMG) [4, 10,11]. The OMG is an organization of over 600 software vendors and users involved in the development of object technology for distributed computing systems. The OMG does not produce any software, only specifications which come from OMG members who respond to Requests for Proposals.

In 1990, the OMG introduced an architecture for inter-operability of object oriented applications called the Object Management Architecture (OMA). The OMA consists of four components: Object Request Broker (ORB), Object Services, Common Facilities, and Application Objects. The OMA object model is a client/server model where the servers are objects that provide services to clients. The clients obtain services by invoking operations on server objects.

The ORB, a key piece of the OMA, is a mechanism that provides transparency of object location, activation, and communication. It also provides interoperability between applications in heterogeneous distributed environments. Object Services, which sit closer to the ORB, provide basic services for using and implementing objects. Common Facilities, which sit closer to the user, provide some generic functions for specific requirements. Unlike Object Services, Common Facilities are optional. Finally, Application Objects are objects specific to certain applications, which may be built from other objects such as Object Services and Common Facilities.

The CORBA specification, introduced in 1991, describes the interfaces and services that ORBs must have; i.e., CORBA is basically the technology adopted for 
ORBs. CORBA provides a clean model where the interface of an object and its underlying implementation are separated; clients do not need to know how or where servers are implemented. Server objects are visible only through interfaces and object references. Interfaces describe the services provided by an object and object references identify objects. An ORB uses object references to identify and locate objects to forward requests to them.

The CORBA specification has several components: ORB Core, Interface Definition Language (IDL), Dynamic Invocation Interface (DII), Interface Repository, and Object Adapters. The CORBA 2 specification, introduced in late 1994, added more components, mainly in inter-ORB interoperability of ORB implementations from different vendors and the Dynamic Skeleton Interface (DSI). The DSI provides a run-time binding mechanism for servers.

The ORB Core handles requests for remote objects. It uses object references to locate objects, activates them (if they are not already active), delivers the request to them, transfers control to them, and finally returns any output values to the client.

IDL is a declarative language for describing the interfaces of CORBA objects with a syntax resembling that of $\mathrm{C}++$. It is a subset of $\mathrm{C}++$ with $\mathrm{C}++$ implementation constructs removed and with extensions for distributed programming. It supports multiple interface inheritance. IDL interfaces contain attributes and operations used to define services provided by objects. An IDL compiler maps IDL constructs into a specific programming language (such as $\mathrm{C}++$ or $\mathrm{C}$ ) based on CORBA language bindings. The compiler generates the client and server code, called client stubs and server skeletons, needed to implement the interface.

In the IDL to $\mathrm{C}++$ mapping, each interface is mapped into a $\mathrm{C}++$ class while each operation is mapped into a $\mathrm{C}++$ member function. Each read-write attribute is mapped into two member functions (get and set) whereas each read-only attribute is mapped into a get function only.

Interface operations may be invoked either statically through the compiler generated stubs or dynamically through DII. The DII is a mechanism for clients to make calls on objects with no compile-time knowledge of their interfaces. It is usually more flexible but more complicated and less efficient than the static invocation interface (through client stubs).

The Interface Repository provides persistent storage for IDL interface declarations. It provides run-time information about the interface properties of objects. The Object Adapters provide the means for object implementations to access ORB services. Among these services are object reference generation, object operation invocation, activation and deactivation of objects, and security. CORBA specifies that a standard adapter called the Basic Object Adapter (BOA) should be provided by every ORB.

Object invocation in CORBA can be done synchronously (blocking), asynchronously (non-blocking), or one-way (best-effort). In a synchronous communication, the sender sends a request and waits until the request either completes or fails. In an asynchronous communication (called deferred synchronous communication in CORBA terminology), the sender sends a request and proceeds with other work (does not wait) but it must check periodically for a response. Asynchronous communication 
is supported under the DII only. In a one-way communication, the sender sends a request and proceeds with other work without checking for a response. Here the receiver does not return a value to the sender.

\section{Orbix}

Orbix is a library based implementation of the CORBA specification from IONA Technologies Ltd [5]. It is implemented mainly by two sets of libraries (client and server libraries) and a daemon, orbixd. The server library can send and receive remote object requests while the client library can only send requests. The daemon needs to run on the server's host side so it can start server processes dynamically. Orbix also has an IDL compiler, Interface Repository and many utilities. The Orix IDL compiler generates a $\mathrm{C}++$ class for an IDL interface to be used by client programs. The Orbix communication classes are implemented using TCP/IP, XDR, and a simple message protocol.

Orbix runs on MS Windows as well as several Unix platforms, including Sun Solaris, SGI IRIX, IBM AIX, HP UX, and DEC Ultrix. The implementation tested is Orbix version 1.3.3.

\section{Parallel Programming System: PVM}

The Parallel Virtual Machine (PVM) is a collection of public-domain system software routines that enables parallel processing on a network of heterogeneous computers as well as parallel computers [2]. It is composed of two parts: a run time system (daemon) that resides on all of the computers participating and a set of user interface primitives that can be incorporated into a $\mathrm{C}$ (or Fortran) code. This includes primitives for process control, message passing, and synchronization between processes running on different machines.

PVM daemons communicate with one another through UDP sockets while a PVM task communicates with its daemon over a TCP connection. Also, PVM tasks can communicate with each other by establishing a direct TCP link between the tasks. Our implementation is based on PVM version 3.3 .10 using a direct TCP link between PVM tasks.

PVM supports both synchronous and asynchronous communication forms. In addition to these point-to-point types of communication, PVM supports group communication such as multicast, to a set of tasks, and broadcast, to a user defined group of tasks.

\section{Test Platforms}

Two platforms were used for this study: a cluster of SGI workstations, called DaVinci, and two SGI workstations connected by an ATM switch, called here the ATM testbed. The DaVinci cluster consists of nine (one front end system and eight compute nodes) SGI Power Challenge machines with MIPS R8000 CPUs. The front end is a four-cpu $75 \mathrm{MHz}$ shared-memory node thus serves as the compile server, file server, user home 
server, and others. The eight compute nodes (four single-cpu $75 \mathrm{MHz}$ nodes, two eight-cpu $90 \mathrm{MHz}$ shared-memory nodes, and two ten-cpu $90 \mathrm{MHz}$ shared-memory nodes) are connected via Ethernet, FDDI, and HiPPI. In addition, all nodes have ATM network interfaces, however, the ATM drivers are not currently ready for use. Each of the single-cpu nodes has 128 Mbytes of memory while each of the multiplecpu shared-memory nodes has either 2 Gbytes or 4 Gbytes of main memory. Only the single-cpu nodes were utilized in this study. All nodes are currently running SGI's IRIX 6.2 operating system, which is a 64 -bit UNIX operating system.

The ATM testbed consists of two SGI Indigo2 machines with a single-cpu 200 $\mathrm{MHz}$ MIPS R4000 processor and 128 Mbytes of main memory. The two machines, each has a Fore ESA-200E ATM OC-3 EISA bus adaptor, are connected directly to each other with no switch in between. The ATM performance is limited by the 80 Mbits/s EISA bus. The two machines are currently running SGI's IRIX 5.3 operating system.

Briefly, Ethernet is a $10 \mathrm{Mbits} / \mathrm{s}$ broadcast bus technology while Fiber Distributed Data Interface (FDDI) is a $100 \mathrm{Mbits} / \mathrm{s}$ fiber optic token ring network. Both Ethernet and FDDI are connectionless networks. The maximum frame size for Ethernet is 1500 bytes while the maximum frame size for FDDI is 4500 bytes. High Performance Parallel Interface (HiPPI) is a point-to-point link that uses twisted-pair copper cables with a maximum length of 25 meters and a transfer rate of $800 \mathrm{Mbits} / \mathrm{s}$. HiPPI is a connection-oriented network that uses variable sized packets up to 64 Kbytes. Asynchronous Transfer Mode (ATM) is a connection-oriented protocol that uses fixed length cells of 53 bytes, with 48 bytes of payload. ATM uses an adaptation layer to frame ATM cells into 9180 byte frames. Both HiPPI and ATM are switched networks while Ethernet and FDDI are shared medium networks. However, both Ethernet and FDDI have switching technology available.

\section{Performance Results: BSD Socket Interface}

Several communication tests were performed on the two platforms using two C programs: ttcp and bench. Both programs use BSD sockets. The ttcp program measures point-to-point data transfer throughput using either TCP or UDP protocols. It uses bulk transfer where the data flows in one direction while the sender sends a prespecified number of messages. It has many options including: message size, socket buffer size, number of messages, and setting TCP_NODELAY (which controls buffering in sending data). In this work, ttcp was chosen to measure throughput using TCP with TCP_NODELAY disabled (by default).

The bench program [7] implements two types of tests: bulk transfer and roundtrip. In a bulk transfer, which is similar to $t t c p$, a number of messages are transferred back to back through the network. When the transfer completes, the receiver sends a single message back to the sender for acknowledgement. In a round-trip test, messages are sent (one at a time) from one machine to another, then echoed back. In this work, the round-trip test was chosen to measure latency with UDP because of the simple nature of the protocol.

The throughput measure (using $t t c p$ ) was conducted for different message sizes 
and socket buffer sizes. The message size was varied (through doubling) from 1 to 64 Kbytes. Two socket buffer sizes ( 8 and 64 Kbytes) were considered, except for HiPPI where 1 Mbytes buffer size was also considered since a previous work [1] showed that HiPPI needs a large buffer to achieve good performance. Each test was run for at least 20 seconds to produce reliable data. All measurements were obtained under conditions of light network traffic. However, we noticed some fluctuations in the timing results.

Figure 1 shows the performance results using BSD sockets for the following networks: Ethernet, FDDI, HiPPI on DaVinci and ATM on the ATM testbed using 8, 64 and 1024 (HiPPI only) Kbytes socket buffer sizes. The best achieved performance results are with HiPPI especially with the 1 Mbytes socket buffer where it outperformed both ATM and FDDI by an order of magnitude and Ethernet by two orders of magnitude. Performance differences between FDDI and ATM are within $30 \%$ where FDDI outperformed ATM for the larger buffer size while the latter outperformed the former for the smaller buffer.

Figure 1 results also show that better performance was achieved using the larger buffer size, except for Ethernet where the $8 \mathrm{~K}$ results outperformed the $64 \mathrm{~K}$ results by over $20 \%$. The impact of the buffer size is more significant for HiPPI where high performance was achieved only using large buffer size (1 Mbytes) and large messages (16 Kbytes and larger). The differences in performance between the $8 \mathrm{~K}$ and $64 \mathrm{~K}$ socket buffer results are more than a factor of two for FDDI while it is less than that for ATM. The impact of the socket buffer size can be attributed to the TCP window size since TCP breaks up the data into segments. Larger window sizes allow the transmission of multiple TCP segments (fill the pipe) before an acknowledgement arrives.

A simple linear regression model was developed for the $t t c p$ results. The model is based on the following equation: $T=b_{0}+b_{1} * m$, where $T$ is the predicted cost for a message of length $m, b_{0}$ is the intercept of the line, and $b_{1}$ is the slope. For round-trip measurements, $b_{0}$ could be considered as the cost of a zero-byte message but for bulk transfer, as in $t t c p, b_{0}$ has no real meaning. The inverse of $b_{1}$ is the maximum achievable throughput, $r_{\max }$, for that test. The goodness of a regression is measured by the coefficient of determination, $R^{2}$. The higher the value of $R^{2}$, the better the regression; for a perfect model $R^{2}$ is 1 .

The regression coefficients $b_{0}$ and $b_{1}$ are estimates for a single test. In order to obtain better estimates, the $90 \%$ confidence intervals for $b_{1}$ and $r_{\max }$ are computed [6]. The meaning of the $90 \%$ confidence intervals for $r_{\text {max }}$, for example, is that we can state with $90 \%$ confidence that the maximum achievable throughput of a network is between two values and the chance of error in this statement is $10 \%$.

Table 1 lists the values of $r_{\max }$, the $90 \%$ confidence intervals for $r_{\max }$, and $R^{2}$ obtained from applying the regression model on the observed results of Figure 1 for the specified buffer sizes. The values of $R^{2}$ show that the predicted results matched the observed results very well. Table 1 also shows the startup latency, $t_{0}$, and the half performance length, $n_{1 / 2}$. The startup latency is the time required to send a message of minimum size and was measured using the round-trip test of the bench program (using an eight-byte message). The half performance length is the message size needed 
Table 1: Network parameters using ttcp/C

\begin{tabular}{|l|r|c|c|r|r|r|}
\hline Network & $\begin{array}{c}r_{\max } \\
(\mathrm{Mbits} / \mathrm{s})\end{array}$ & $\begin{array}{c}r_{\max } 90 \% \text { confidence } \\
\text { intervals }(\text { Mbits } / \mathrm{s})\end{array}$ & $R^{2}$ & $\begin{array}{c}n_{1 / 2} \\
(\mathrm{Bytes})\end{array}$ & $\begin{array}{c}t_{0} \\
(\mu \mathrm{sec})\end{array}$ & $\begin{array}{c}\text { buf size } \\
(\text { Kbytes })\end{array}$ \\
\hline Ethernet & 8.3 & $8.3,8.3$ & 1.000 & 14 & 550 & 8 \\
FDDI & 89.4 & $88.5,90.4$ & 1.000 & 740 & 561 & 64 \\
HiPPI & 716.5 & $710.2,722.8$ & 1.000 & 9826 & 593 & 1024 \\
ATM & 70.0 & $69.6,70.4$ & 1.000 & 364 & 360 & 64 \\
\hline
\end{tabular}

to achieve half of $r_{\max }$ and is computed from Figure 1 with some approximation since data was not collected for all message sizes.

The results of Figure 1 and Table 1 show that Ethernet, FDDI, HiPPI, and ATM have achieved over $80 \%$ of their peak rates provided that the limiting factor for the ATM performance is the EISA bus.

The $n_{1 / 2}$ measure shows that Ethernet is very efficient even with very small messages due to the fact that Ethernet is a mature technology and its software has been well optimized. On the other hand, HiPPI needs about a $10 \mathrm{Kbyte}$ message to achieve half of its maximum achievable rate. The $t_{0}$ results showed that latency is the smallest for ATM while it is about the same for the other networks.

\section{Performance Results: CORBA/Orbix}

Performance of Orbix version 1.3.3 was measured using two Orbix programs: ttcp/Orbix and timer. The program ttcp/Orbix [9] is a modified version of $t t c p$ which replaces all $\mathrm{C}$ socket calls with stubs and skeletons generated from two CORBA IDL definitions for messages: sequence and string. Unbounded IDL sequences are basically dynamically sized arrays while a string is a sequence of char. This program, like ttcp, measures the bulk transfer rate of a network for different message sizes and socket buffer sizes, since Orbix provides a mechanism to change the buffer size through a user-defined call back function. For more details about the code, see [9].

The timer program, originally written by Mokkapati [8], measures both bulk transfer and round-trip rates for different data types. This program was modified to measure the round-trip time for a zero-length message and throughput for variable length data types.

Figure 2 shows the performance results for the four networks under Orbix using 8 and $64 \mathrm{Kbytes}$ socket buffer sizes. The message size was varied (through doubling) from 1 to 64 Kbytes. All results are for messages of type sequence since sequence outperformed string for all our tests. This can be attributed to differences in their IDL to $\mathrm{C}++$ mappings since, unlike the IDL sequence mapping, the string mapping does not include a length field so strlen is done on both sides during marshalling and demarshalling data.

Figure 2 results show that, similar to the BSD sockets results, performance with the 64 Kbytes buffer is consistently higher than using the 8 Kbytes buffer for all networks except Ethernet where the latter outperformed the former by more than 
Table 2: Network parameters using ttcp/Orbix (64 Kbytes buffer size)

\begin{tabular}{|l|r|c|r|r|r|}
\hline Network & $\begin{array}{c}r_{\max } \\
(\mathrm{Mbits} / \mathrm{s})\end{array}$ & $\begin{array}{c}r_{\max } 90 \% \text { confidence } \\
\text { intervals }(\text { Mbits } / \mathrm{s})\end{array}$ & $R^{2}$ & $\begin{array}{c}n_{1 / 2} \\
(\text { Bytes })\end{array}$ & $\begin{array}{c}t_{0} \\
(\mu \mathrm{sec})\end{array}$ \\
\hline Ethernet & 6.3 & $6.3,6.3$ & 1.000 & 206 & 1621 \\
FDDI & 77.3 & $73.3,81.8$ & 0.996 & 5763 & 1628 \\
HiPPI & 130.3 & $119.3,143.5$ & 0.993 & 7811 & 1672 \\
ATM & 54.4 & $50.6,58.7$ & 0.993 & 3670 & 1166 \\
\hline
\end{tabular}

$20 \%$. The HiPPI performance under Orbix peaked at around $100 \mathrm{Mbits} / \mathrm{s}$ using a 32 Kbytes message and a 64 Kbytes buffer and then dropped significantly for larger messages and buffer sizes (not shown in the figure). As in BSD sockets, HiPPI outperformed other networks under Orbix but by smaller factors than with BSD sockets. Also FDDI outperformed ATM by about the same margin as for BSD sockets using a 64 Kbytes buffer.

A linear regression model, similar to the BSD sockets model, was developed for the Orbix results. The results of applying the model for the 64 Kbytes buffer are listed in Table 2. Also, the startup latency and the half performance length, defined in the previous section, are given in the table. The latency was computed using the round-trip test of the timer program (zero and one byte messages). The half performance length was computed from Figure 2 with some approximation.

The results of Figure 2 and Table 2 show that Ethernet, FDDI, and ATM under Orbix achieved over $70 \%$ of their peak rates while HiPPI achieved only $16 \%$ of its peak rate. The problem with HiPPI is that it needs large buffer sizes to run efficiently and that could not be achieved under Orbix. Latency under Orbix is about three times that of the BSD sockets for all networks. Also, the half performance length is higher under Orbix than using the BSD sockets.

A comparison between Orbix and BSD sockets results for the same socket buffer size shows a drop in performance of up to a factor of three depending on the message and socket buffer sizes. The values of $r_{\max }$ for FDDI, HiPPI, and ATM under Orbix are about $70 \%$ to $85 \%$ of their values under BSD sockets for the same socket buffer size whereas the differences are insignificant for Ethernet.

The CORBA overhead can be attributed to many factors, including: data copying, presentation layer conversion, demultiplexing, and memory allocation [9]. The UNIX profiler prof was used to give some insight to the sources of CORBA overheads. The prof program gives some estimates of the amount of time spent in every function of a program. Even though the prof results were not conclusive and some abnormality was observed, it was noticed that a reasonable percentage of the execution time was spent in memcpy. This shows that there were many memory copy operations and the IDL stubs and sequences may copy data several times.

Table 3 shows performance variations under Orbix for transferring sequences of struct and char using the timer program. In IDL, as in C and $\mathrm{C}++$, a struct data type allows related data types to be packaged together. These results show that the use of 
Table 3: Network performance (in Mbits/s) using Orbix (16 Kbytes message size)

\begin{tabular}{|l|r|c|r|r|}
\hline \multirow{2}{*}{ Network } & \multicolumn{2}{|c|}{ bulk transfer } & \multicolumn{2}{c|}{ round-trip } \\
\cline { 2 - 5 } & char & struct & char & struct \\
\hline Ethernet & 6.7 & 5.4 & 7.4 & 4.4 \\
FDDI & 84.4 & 15.6 & 41.1 & 9.5 \\
HiPPI & 84.0 & 14.3 & 65.7 & 11.0 \\
ATM & 60.0 & 18.0 & 39.6 & 11.5 \\
\hline
\end{tabular}

Table 4: Network parameters using ring/PVM.

\begin{tabular}{|l|r|c|r|r|r|}
\hline Network & $\begin{array}{c}r_{\max } \\
(\text { Mbits/s })\end{array}$ & $\begin{array}{c}r_{\max } 90 \% \text { confidence } \\
\text { intervals (Mbits/s) }\end{array}$ & $R^{2}$ & $\begin{array}{c}n_{1 / 2} \\
(\text { Bytes })\end{array}$ & $\begin{array}{c}t_{0} \\
(\mu \mathrm{sec})\end{array}$ \\
\hline Ethernet & 8.4 & $8.4,8.4$ & 1.000 & 544 & 830 \\
FDDI & 70.0 & $66.5,74.0$ & 0.997 & 12098 & 942 \\
HiPPI & 102.7 & $94.0,113.1$ & 0.990 & 9278 & 854 \\
ATM & 26.8 & $25.6,28.2$ & 0.987 & 1419 & 602 \\
\hline
\end{tabular}

struct, instead of char, caused a significant performance drop for all networks using both bulk transfer and round-trip communications. This drop is due to the overhead in marshalling and demarshalling structs under Orbix. This was also observed in [3] using SPARCstations.

\section{Performance Results: PVM}

Performance of the four networks under the PVM message passing library version 3.3.10 was measured using a $C$ program, called ring; see [1] for more details. In ring, the processors form a ring where each processor receives a message of prescribed length from a previous processor and sends the same message to the next processor. Only one message goes around the ring at any given time. This program measures point-to-point performance and latency of a network.

Figure 3 shows the performance results for the four networks under PVM for message sizes ranging between 1 and 64 Kbytes. Under PVM, HiPPI outperformed the other networks but both HiPPI and ATM achieved only small fractions of their peak rates. The ATM performance under PVM peaked at around $25 \mathrm{Mbits} / \mathrm{s}$ using a 16 Kbytes message and then dropped significantly for larger messages (not shown in the figure).

A linear regression model, similar to the BSD sockets and Orbix models, was developed for the PVM results. Table 4 lists the results of applying the model on the PVM results. The half performance length is relatively long for FDDI and HiPPI compared to Ethernet and ATM. Latency under PVM is less than $1 \mathrm{msec}$ for all networks. It is below the latency under Orbix. 
Performance of PVM is slightly below Orbix for FDDI and HiPPI while it is significantly below Orbix performance with ATM. One of the reasons for these differences is the ability to change the socket buffer size under Orbix while it is hard to change it under PVM.

The PVM overhead can be attributed to many factors, including: buffer managements, connection management, and state maintenance. These overheads are more apparent in new networks (such as HiPPI and ATM) than in traditional networks.

\section{Concluding Remarks}

High-level network programming interfaces, such as CORBA, provide many advantages over low-level, non-typesafe programming interfaces, such as the BSD sockets. Among these advantages are extensibility, maintainability, and reusability. These high-level interfaces have been traditionally less efficient than the low-level interfaces, especially on high-speed networks. This study showed that might still be the case but reasonable performance can be achieved. Also, users might be willing to accept certain performance penalty given all the benefits they are gaining from using these high-level interfaces. However, users have to be aware of some of performance restrictions that are associated with the use of certain data types. For example, the use of IDL strings as well as structs carries some performance penalties compared to the use of sequences and chars.

Performance differences between CORBA and PVM are not quite significant. The choice between the two depends on many other factors including the programming model, whether it is procedure-oriented or object-oriented. Their performance on high-speed networks suffer due to software overheads. However, performance of highlevel interfaces is not fixed - it keeps improving. There have been many studies in how to improve communication software, such as compiler optimization techniques and using light-weight protocols. Some of these optimizations are being utilized in commercial network interfaces and operating systems.

The emphasis of this study is performance at the communication level. More work needs to be done using real applications to have a better understanding of the limiting factors of communication software at the application level.

\section{Acknowledgment}

I would like to thank Sandra Johan of NASA Ames and Noemi Berry of MRJ, Inc. for many technical discussions about CORBA and ATM. I am also thankful to IONA Technologies Ltd. for providing me with an evaluation copy of Orbix 1.3.3.

\section{References}

[1] Fatoohi, R., Performance Evaluation of Communication Networks for Distributed Computing, in the Proceedings of the Fourth International Conference on Computer Communications and Networks (Las Vegas, 1995), IEEE Computer Society Press,

pp. 456 459. 
Also in URL: "http://www.nas.nasa.gov/NAS/TechReports/NASreports/NAS95-009/NAS-95-009.html"

[2] Geist, A., Beguelin, A., Dongarra, J., Jiang, W., Manchek, R., and Sunderam, V., PVM 3 User's Guide and Reference Manual. Report ORNL/TM12187, Oak Ridge National Lab., Oak Ridge, TN, September 1994. Also in URL: "http://www.epm.ornl.gov/pvm"

[3] Gokhale, A., Schmidt, C., Cytron, R., and Varghese, G. Compiler Optimization Techniques for Improving Performance of Distributed Object Computing Frameworks over High-speed ATM Networks, In the Software Technology Conference, (Salt Lake City, 1996). Also in URL: "http://www.cs.wustl.edu/ " schmidt"

[4] Information Resources on CORBA and the OMG, URL: "http://www.acl.lanl.gov/CORBA"

[5] IONA Technologies, The Orbix Architecture, IONA Technologies Ltd., Dublin, Ireland, January $1995 . \quad$ Also in ${ }^{\circ}$ URL: "http://www.iona.com:8000/www/Orbix/arch/Summary.html"

[6] Jain, R., The Art of Computer Systems Performance Analysis, Wiley \& Sons, Inc., 1991.

[7] Keeton, K., Anderson, T., and Patterson, D., LogP Quantified: The Case for Low-Overhead Local Area Networks. In Proceedings of HOT Interconnects III, Stanford, CA, August 1995.

[8] Mokkapati, R., Post Modern Computing, Mountain View CA 94043.

[9] Schmidt, C., Harrison, T., and Al-Shaer, E., Object-Oriented Components for High-speed Network Programming, In the Proceedings of the Conference on Object-Oriented Technologies, (Monterey, 1995), USENIX. Also in URL: "http://www.cs.wustl.edu/ schmidt"

[10] Vinoski, S., Distributed Object Computing With CORBA, C++ Reports Magazine, July/August 1993.

[11] Yang, Z., and Duddy, K., Distributed Object Computing with CORBA, Technical Report 23, Distributed Systems Technology Centre, The University of Queensland, QLD 4072 Australia, June 1995. Also in URL: "http://www.dstc.edu.au/AU/research_news/omg/corba.html" 


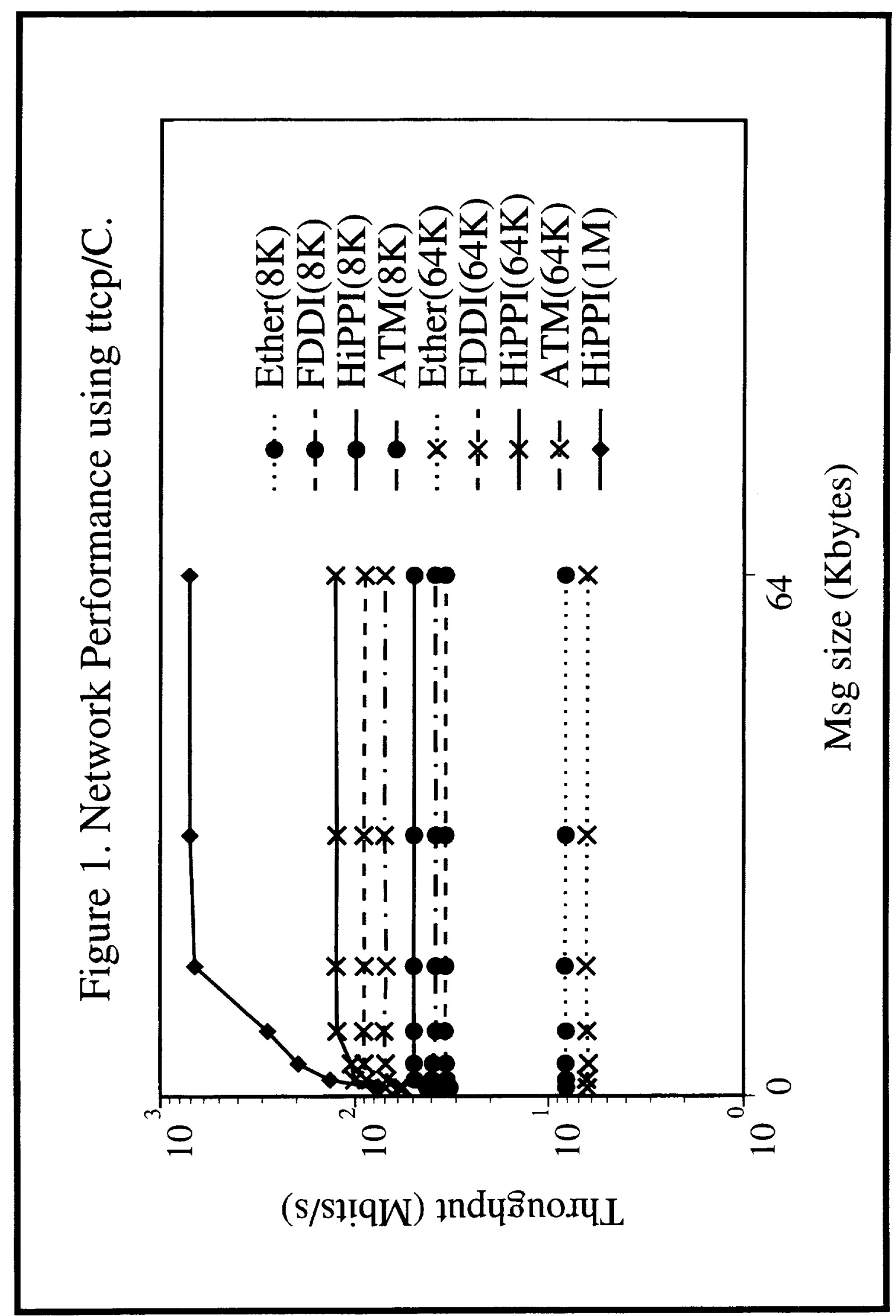




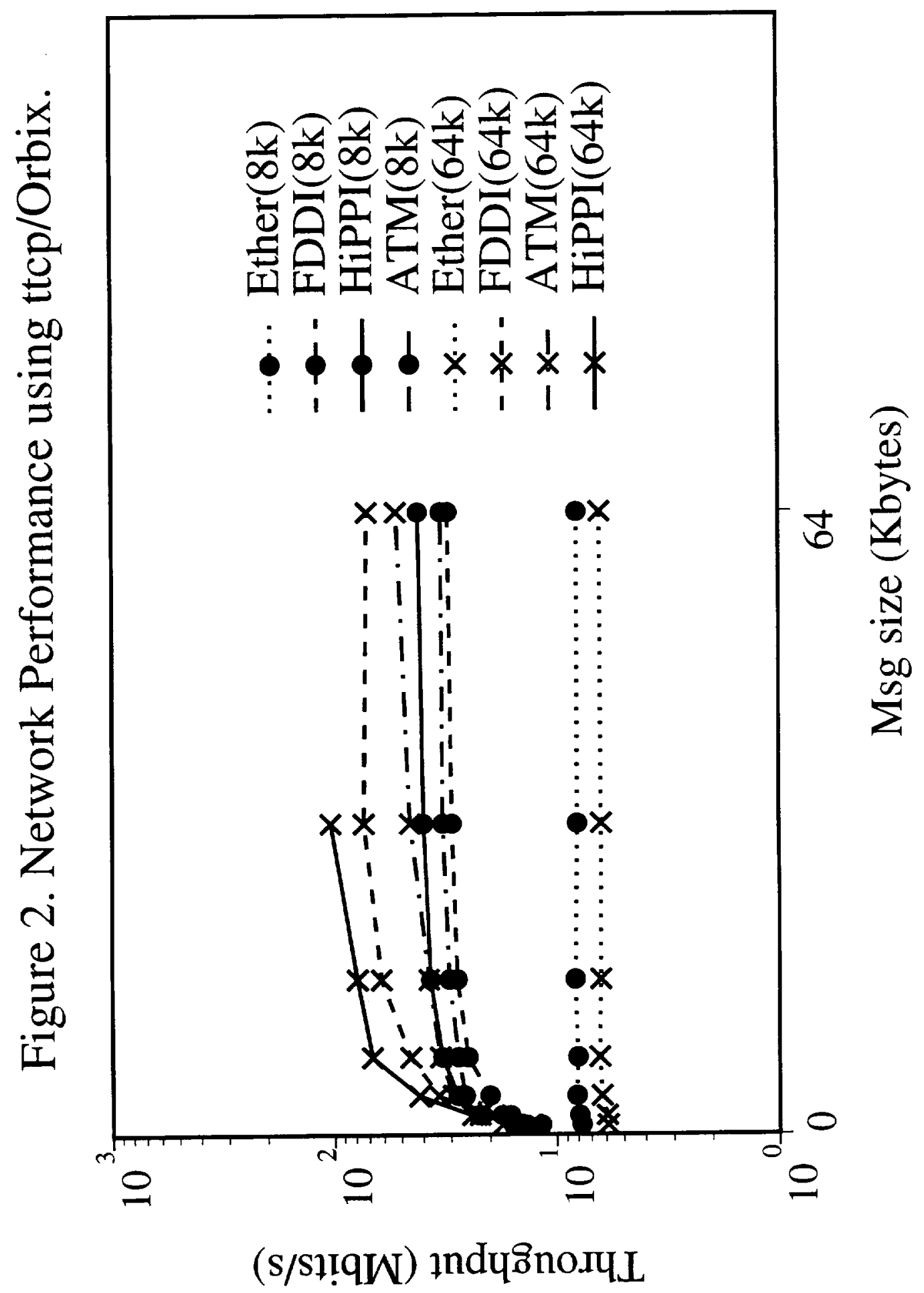




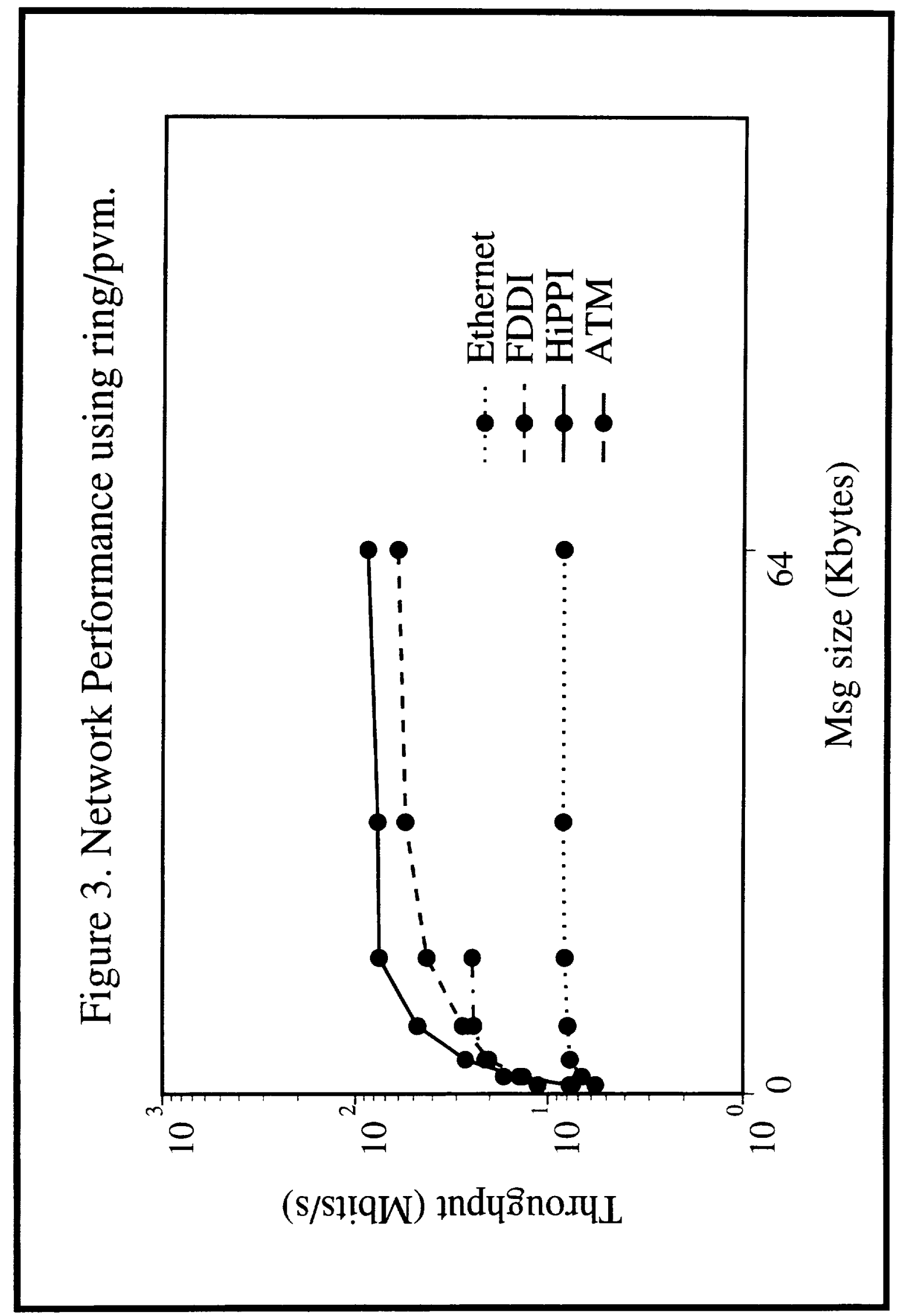




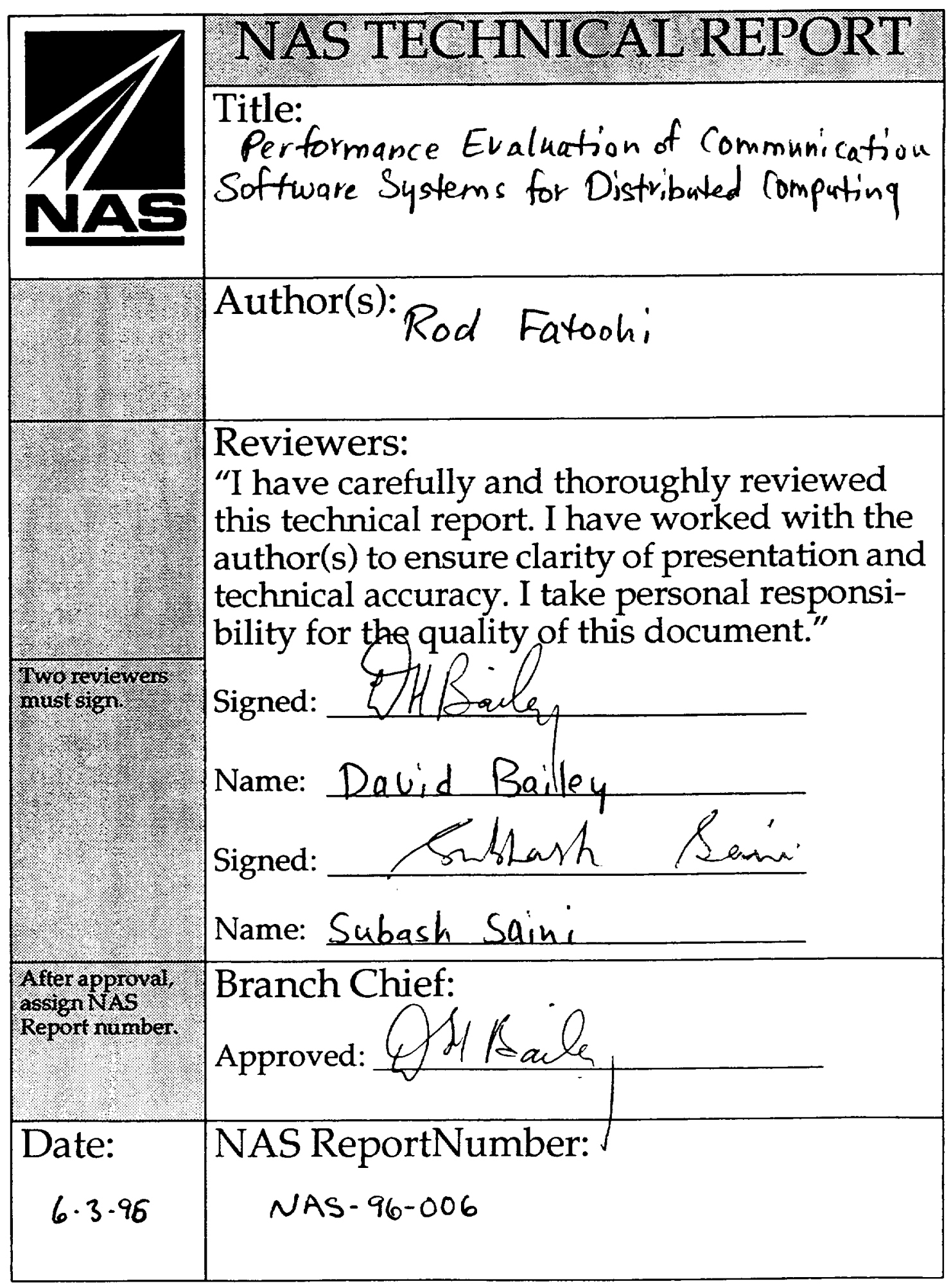


